\title{
High-Pressure Structural and Equation of State Study of Atacamite, a Copper Hydroxychloride Mineral
}

\author{
N Ross ${ }^{1}$, J Zhao ${ }^{2}$ \\ ${ }^{1}$ Geosciences, Virginia Tech, Blacksburg, VA, ${ }^{2}$ Virginia Tech, Blacksburg, VA \\ nross@vt.edu
}

We studied the effect of pressure on atacamite, $\mathrm{Cu} 2 \mathrm{Cl}(\mathrm{OH}) 3$, to $8.79 \mathrm{GPa}$ using single-crystal X-ray diffraction. Atacamite, crystallizes in orthorhombic space group Pnma with $\mathrm{a}=6.0323(1) \AA, \mathrm{b}=6.8672(2) \AA, \mathrm{c}=9.1207(5)$ $\AA, \mathrm{V}=377.018(8) \AA^{\wedge} 3$, and $\mathrm{Z}=4$. The $(\mathrm{OH}) 3 \mathrm{Cl}$ group forms a tetrahedron, $\mathrm{O} 3 \mathrm{Cl}$, with two $\mathrm{H}(2)$ atoms positioned inside its $\mathrm{O}(2) \ldots \mathrm{Cl}$ edges, and one $\mathrm{H}(1)$ just outside its $\mathrm{O}(1) \ldots \mathrm{Cl}$ edge [1]. The $\mathrm{Cu}$ atoms reside in distorted octahedral (4+2)-coordination sites: $\mathrm{Cu}(1)$ is bonded to four hydroxyl groups and two $\mathrm{Cl}$ atoms while $\mathrm{Cu}(2)$ is bonded to five hydroxyl groups and one $\mathrm{Cl}$ atom. These $\mathrm{Cu}$-octahedra are edge-linked as in the spinel structure [1]. The structure is stable throughout the pressure range studied but there is a distinct change in the equation of sate of atacamite at 3.5 GPa reflected in the change of the isothermal bulk modulus, $\mathrm{K}$, as a function of pressure (Fig. 1). A 3rd-order Birch-Murnaghan equation of state fit to the P-V data up to $3.44 \mathrm{GPa}$ yielded $\mathrm{K}=79.8(9)$ GPa with a very low $\mathrm{dK} / \mathrm{dP}=0.5(5)$. Above $3.5 \mathrm{GPa}$, a 3rd-order Birch-Murnaghan equation of state fit to the data yielded $\mathrm{K}=74.0(5) \mathrm{GPa}$ and $\mathrm{dK} / \mathrm{dP}=3.6(1)$. The structural changes that occur at $3.5 \mathrm{GPa}$ will be discussed in detail in the presentation. [1] Parise and Hyde (1986) Acta Cryst, C42: 1277-1280

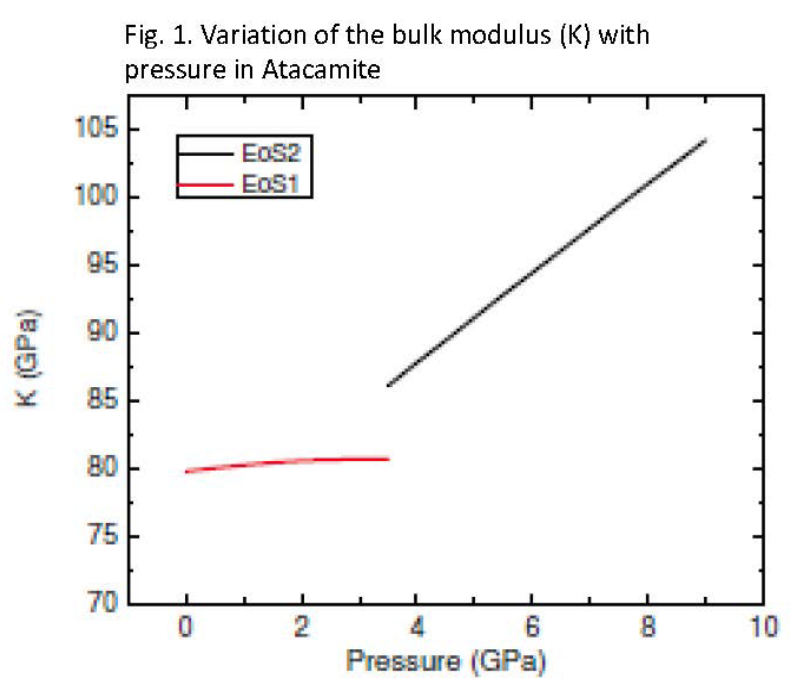

Figure 1.

Acta Cryst. (2020). A76, a115 